\title{
Muerte súbita intrahospitalaria luego de infarto agudo de miocardio en Cuba en los últimos tres años. Análisis de registros institucionales
}

\section{Sudden intra-hospital death after acute myocardial infarction in Cuba in the last three years. Analysis of institutional records}

\section{Miguel A. Rodríguez-Ramos ${ }^{1}$ y Maikel Santos-Medina²}

${ }^{1}$ Unidad de Cuidados Intensivos Coronarios, Hospital General Docente Camilo Cienfuegos, Universidad de Ciencias Médicas Faustino Pérez Hernández, Sancti-Spiritus; ${ }^{2}$ Unidad de Cuidados Intensivos Coronarios, Hospital General Docente Ernesto Guevara, Universidad de Ciencias Médicas Zoilo Marinello Vidaurreta, Las Tunas. Cuba

\section{Resumen}

Objetivo: Analizar el comportamiento de posibles causas predisponentes de muerte súbita (MS) intrahospitalaria luego de un infarto agudo de miocardio (IMA) en registros cubanos. Material y método: Se realizó una búsqueda de registros clínicos de pacientes con IMA en Cuba en las bases de datos de revistas nacionales, Scientific Library On-line (ScieLO) y Medline. Se priorizaron los artículos publicados desde 2016 para ser incluidos. Se definió como muerte súbita aquélla secundaria a arritmias ventriculares malignas (TV y FV), así como los pacientes con rotura cardíaca y actividad eléctrica sin pulso o asistolia como forma de presentación. Con posterioridad se evaluó la relación de este parámetro con la aparición de muerte súbita en 710 pacientes del Registro de Síndromes Coronarios Agudos (RESCUE). Resultados: En el contexto extrahospitalario, más de la mitad de las muertes súbitas cardíacas son secundarias a un infarto agudo de miocardio. En el hospital, la mortalidad en Cuba por IMA es homogénea. Sólo los centros con intervencionismo coronario escapan a este fenómeno. Aunque no del todo letales, las arritmias ventriculares malignas se relacionan con un peor pronóstico y su prevalencia no es homogénea en los registros revisados. Conclusiones: La muerte súbita luego de infarto agudo de miocardio será aún en Cuba una de las principales causas de muerte en los pacientes de fase aguda.

Palabras clave: Muerte súbita cardíaca. Mortalidad hospitalaria. Infarto de miocardio. Infarto de miocardio agudo con elevación del segmento ST.

\begin{abstract}
Objective: To analyze possible predisposing causes of in hospital sudden cardiac death (SCD) after an acute myocardial infarction (IMA) in Cuban registries. Material and methods: A search of clinical records of patients with IMA in Cuba was performed in the databases of national journals, Scientific Library On-line and Medline. Those articles published since 2016 were prioritized for inclusion. Sudden death is defined as that secondary to malignant ventricular arrhythmias (ventricular tachycardia, ventricular fibrillation) as well as patients with cardiac rupture with pulseless electrical activity or asystole as a form of presentation. Subsequently, the relationship of this parameter with the occurrence of sudden death was evalua-
\end{abstract}

Correspondencia:

Miguel A. Rodríguez-Ramos

E-mail: mialero@infomed.sld.cu
Disponible en internet: $14-09-2020$ Arch Cardiol Mex. 2020;90(3):341-346 www.archivoscardiologia.com 1405-9940 / @ 2020 Instituto Nacional de Cardiología Ignacio Chávez. Publicado por Permanyer. Este es un artículo open access bajo la licencia CC BY-NC-ND (http://creativecommons.org/licenses/by-nc-nd/4.0/). 
ted in 710 patients from the Registry of Acute Coronary Syndromes (RESCUE). Results: In the out-of-hospital setting, more than half of SCD are secondary to an IMA. Once in the hospital, mortality in Cuba from IMA is homogeneous. Only centers with coronary interventionism escape this phenomenon. Although not totally lethal, the presence of malignant ventricular arrhythmias is associated with a worse prognosis and its prevalence is not homogeneous in the reviewed records. Conclusions: Sudden death after IMA will continue to be one of the main causes of death of patients in the acute phase in Cuba.

Key words: Sudden cardiac death. Hospital mortality. Myocardial infarction. ST segment elevation IMA.

\section{Introducción}

Las enfermedades cardíacas constituyen una importante causa de muerte en países desarrollados y en vías de desarrollo ${ }^{1}$. Gran parte de éstas ocurre en pacientes sin antecedentes patológicos y, aunque se registra un descenso gradual de la prevalencia, la tasa de mortalidad por algunas causas específicas continúa estable².

Se ha señalado que el $75 \%$ de las muertes extrahospitalarias catalogadas como súbitas se debe a enfermedades de arterias coronarias. Éstas incluían a la taquicardia ventricular como causa del suceso final en $75 \%$ de los casos $^{3}$. En la actualidad, debido a medidas de reducción de la incidencia, prevención primaria y secundaria, esta cifra ha descendido, lo que ha propiciado otras formas de paro cardíaco, como asistolia y actividad eléctrica sin pulso ${ }^{4}$.

En el contexto intrahospitalario, este fenómeno de reducción no ha sido notorio y la taquicardia ventricular y la fibrilación ventricular son aún causas principales de paro cardíaco, razón por la cual las tasas de rescate son elevadas en el ámbito extrahospitalario ${ }^{5}$.

Aunque existen en Cuba tres redes cardioquirúrgicas con posibilidad de intervencionismo coronario y quirúrgico en los centros regentes, éstas se encuentran activas (24/7) pocas veces para la recepción de pacientes con síndromes coronarios agudos (SCA), excepto quizás la del Instituto de Cardiología y Cirugía Cardiovascular. En consecuencia, son los hospitales de atención secundaria los rectores de las redes provinciales y sectoriales de atención a estos pacientes, tratados en su mayoría con trombolíticos, y no con intervencionismo coronario como se sugiere en las guías internacionales.

Este trabajo tiene como objetivo analizar la prevalencia de causas predisponentes de muerte súbita (MS) intrahospitalaria luego de un infarto agudo de miocardio (IMA) en registros cubanos.

\section{Método}

Para identificar los artículos publicados en los últimos tres años en Cuba sobre registros hospitalarios de IMA se consultaron la base de datos Scientific Literature On Line (ScieLO) y los archivos de CorSalud y de la Revista Cubana de Cardiología y Cirugía Cardiovascular (RCCCCV). Se revisaron todos los descriptores que en ScieLO aparecen sugeridos con las palabras clave Infarto + Miocardio. Se realizó la misma operación con "Muerte + Súbita" o "Cardiovascular" o "Cardíaca".

Se concedió prioridad a los informes de menos de tres años de publicación o a los que estuvieran en proceso editorial en ese momento. Se descargó por completo el número de CorSalud dedicado al episodio de muerte súbita en 2016, así como a los registros epidemiológicos del Grupo de Investigación de Muerte Súbita (GIMUS) en Cuba.

Se empleó asimismo el motor de búsqueda de Medline para localizar algún informe nacional en las revistas principales. Los motores de búsqueda de información de revistas no incluidas en ScieLO, PUBMED y SCOPUS, con excepción de RCCCCV, no se utilizaron $y$, aunque se reconoce que pueden existir informes de cierta importancia en éstas, se excluyeron.

El siguiente es el flujograma llevado a cabo. Búsqueda en ScielO Cuba: "Infarto de miocardio": 7 resultados; "Infarto del miocardio": 51; "Infarto del miocardio agudo": 2; "Infarto de miocardio agudo": 4; "Infartos del miocardio": 1; "Muerte cardíaca": 1; "Muerte cardíaca súbita": 3; "Muerte súbita": 23; "Muerte súbita cardíaca": 1; "Muerte súbita cardiovascular": 5; "Infarto agudo del miocardio": 5; "Infarto agudo del miocardio": 39; "Infarto agudo miocárdico": 1; "Infarto miocárdico": 1. Búsqueda en ScieLO regional: Muerte súbita en Cuba": 16 resultados superpuestos, 3 nuevos resultados; Infarto miocardio en Cuba": 28 resultados superpuestos, 3 nuevos resultados. Búsqueda en PUBMED de "Sudden cardiac death in Cuba": 19 resultados.

Definiciones. La muerte súbita se definió como la secundaria a arritmias ventriculares malignas (TV y FV), así como los pacientes con rotura cardíaca y actividad eléctrica sin pulso o asistolia como forma de presentación. En los artículos que tengan un enfoque distinto a éste, se informa debidamente y los resultados 
del análisis se proporcionan con base en la definición anterior, cuando sea posible.

Datos. Desde 2014 se recogen en la sala de cardiología del Hospital Camilo Cienfuegos datos de pacientes ingresados por síndrome coronario agudo. Las especificaciones básicas de este registro se han publicado con anterioridad. En cada epígrafe se trabaja con la totalidad de los pacientes incluidos hasta el 31 de diciembre de 2017: 387 sujetos con infarto de miocardio agudo con elevación de ST (IMAEST) y 323 con infarto de miocardio agudo sin elevación de ST (IMASEST). La nula aplicación de intervencionismo coronario en la mayoría de los hospitales de Cuba hace imposible realizar un análisis anatómico-hemodinámico del fenómeno de la muerte súbita luego del infarto de miocardio agudo.

Estadística. Se utilizó una base de datos creada con el paquete estadístico SPSS v.17.0 para Windows. Se comprobó la distribución normal y homogénea de la muestra ( $p>0.05$ ), lo que permitió realizar pruebas paramétricas. Se realizó una distribución de frecuencias de las variables numéricas en estudio y comparaciones de medias entre muestras relacionadas e independientes. Para comprobar la fortaleza de relación entre variables cualitativas se empleó la prueba no paramétrica ji cuadrada de Pearson y, ante situaciones en que más del $20 \%$ de las frecuencias esperadas presentaron valores menores de 5 , se utilizó la prueba exacta de Fisher. Para comparar las medias de variables cuantitativas se empleó el estadígrafo t de Student para muestras independientes. La validación estadística de los resultados de la investigación adoptó un nivel de significación del 95\% $(p<0.05)$ para los grados de libertad previamente fijados en cada una de las circunstancias presentadas. Para la relación entre las variables dicotómicas y la aparición de complicaciones, mortalidad (MS), o ambas, así como mortalidad aislada, se calculó la razón de momios (odds ratio), y se estableció un límite de confianza de $95 \%$, con una diferencia estadística si $p<0.05$.

La investigación recibió aprobación del Comité de Ética y el Consejo Científico del hospital y, aunque no se publicaron datos de identificación de los pacientes, se respetó su confidencialidad durante su manejo.

\section{Muerte súbita extrahospitalaria por infarto de miocardio agudo}

Hasta $15 \%$ de los pacientes con SCA se presenta como MS y es causa del $30 \%$ de las muertes por $\mathrm{SCA}^{6,7}$. Su incidencia es la misma para pacientes con infarto de miocardio agudo con elevación de ST (IMAEST) e infarto de miocardio agudo sin elevación de ST (IMASEST), aunque desciende hasta la mitad en individuos con angina inestable ${ }^{8}$.

En varias series foráneas, entre 6 y $7.5 \%$ de los pacientes presentó paro cardiorrespiratorio antes del primer contacto con los servicios de salud. En las guías clínicas de la European Society of Cardiology, el valor establecido es del 7\%,8.

En Cuba, la mortalidad extrahospitalaria de pacientes con IMA sin atención médica es tan elevada como $30 \%{ }^{9}$. En este grupo se encuentran los individuos con arritmias complejas y también los enfermos con choque cardiogénico, roturas cardíacas y otras causas de muerte súbita y no súbita. No obstante, esta estadística varía en grado considerable porque los registros extrahospitalarios de este fenómeno son poco frecuentes y están sesgados en su mayoría.

En este apartado, el Grupo de Investigación de Muerte Súbita es líder en investigaciones en el tema en Cuba. En una serie de 474 fallecidos por muerte súbita cardíaca $^{10}$, la prevalencia de ésta en relación con el total de muertes fuera del hospital osciló entre $8.0 \%$ y $12.1 \%$. En $58.4 \%$ de estos sujetos se confirmó un IMA como causa de muerte. En otro de sus informes, de 1,000 decesos súbitos durante 11 años, 247 se catalogaron como muerte súbita cardíaca ${ }^{11}$, y en ellos se constataron lesiones ateroscleróticas del árbol coronario en la totalidad de los casos y trombo coronario en $134(63.5 \%)$.

\section{Arritmias ventriculares en el contexto hospitalario e infarto agudo de miocardio}

\section{Análisis de registros}

En cuanto a la presencia de parada cardiorrespiratoria (PCR) en pacientes que alcanzan un centro secundario de atención los datos son más precisos. En un informe del Hospital Hermanos Ameijeiras (HHA) ${ }^{12}$, de 465 pacientes ingresados con IMA, desde enero de 2007 hasta enero de 2016, las arritmias de tipo ventricular (TV o FV) fueron mucho más frecuentes en pacientes fallecidos ( $58 \%$ vs. $8 \%, p=0.001$ ), a pesar de declararse que las arritmias se presentaron en la misma cantidad de pacientes egresados vivos o fallecidos (63/343 vs. 62/79, respectivamente). Aun así, su incidencia fue baja en realidad (14\%). Asimismo, se observó que los pacientes con IMASEST presentaron mayor relación con la MS que los individuos con IMAEST ( $p$ $=0.001$ ), aunque no se registraron en tablas los datos 
para inferir esta conclusión o para repetir un análisis similar en la muestra del RESCUE.

En un registro del Hospital Militar Central ${ }^{13}$ no se identificó una relación entre la aparición de arritmias y la muerte en los 273 pacientes ingresados entre 2013 y 2016 ( $p=0.2$ ). En sus datos, 258 vivos y 14 fallecidos, se advierte que ésta no constituye en verdad un factor predisponente de mal pronóstico ( $5.42 \%$ vs. $14.3 \%)$, a pesar de que los pacientes muertos con arritmias casi tripliquen el número de aquellos que no las presentaron y egresaron vivos. Cabe señalar que en este informe no se especifica el tipo de arritmia que tuvieron los pacientes incluidos y que esto pudo modificar los resultados obtenidos.

Sin embargo, en un registro del CardioCentro Ernesto Guevara $^{14}$, de octubre de 2012 a diciembre de 2014, que incluyó a 86 pacientes, se describe una frecuencia de arritmias letales (11 pacientes, 12.8\%) cercana a la notificada en el plano internacional. Los pacientes que sufrieron infarto anterior y FV ( 5 en total) tuvieron peor pronóstico respecto de quienes experimentaron infarto inferior y FV (2 en total), sin diferencias entre los grupos de tratamiento otorgados $(5.81 \%$ vs. $2.32 \%, p=0.23)$. A pesar de la consonancia con informes foráneos, se señala que estos pacientes recibieron tratamiento de reperfusión farmacológica y que su ausencia constituía un criterio de exclusión para su inclusión.

Otra notificación de este mismo centro ${ }^{15}$, con una muestra más inclusiva de 118 pacientes, sin fecha especificada, con $6.8 \%$ de arritmias malignas, concluyó que el índice leucoglucémico con valor de corte en 1,235 mostró una sensibilidad del $75 \%$ y una especificidad de $52.6 \%$ para el diagnóstico de éstas.

Asimismo, el índice polimorfo/linfocitos ${ }^{*}$ glucemia (PLG), con punto de corte de 14.8, mostró sensibilidad y especificidad de $87.5 \%$ y $61.8 \%$. A pesar de esta relación, que utiliza herramientas al alcance de centros de países subdesarrollados, muy pocos trabajos con esta metodología se informan en Cuba.

Además, es notable la diferencia de frecuencia de presentación de arritmias ventriculares en estas cohortes prevenientes del mismo centro, y con muy escasa diferencia temporal entre ellas.

Similar incidencia se refiere en el registro de la Unidad de Cuidados Intensivos del Hospital "Dr. Juan Bruno Zayas Alfonso" de Santiago de Cuba ${ }^{16}$. En seis años de estudio sólo se incluyó a 135 pacientes (1.8 paciente por mes de estudio). Debido a la metodología seguida para presentar los datos, no se comunicaron algunos resultados de interés. La frecuencia de aparición de arritmias ventriculares fue de $4.44 \%$ en la población estudiada. No obstante, se obtienen diferencias entre la aparición de éstas y el estado al egreso: $1.68 \%$ vs. $25 \%$ en vivos y fallecidos, respectivamente $(p=0.001)$; en este estudio, presentar arritmias ventriculares incrementaba el riesgo de muerte al menos 3.22 veces.

En ninguno de los informes analizados se observan más de cinco pacientes por mes de estudio. En el caso específico del RESCUE, el IMAEST representa casi 10 pacientes por mes de estudio. Una posible explicación de esta baja prevalencia de ingresos puede ser el sitio de recolección de datos. Los pacientes trasladados 0 ingresados en salas de cuidados intensivos o centros terciarios son casi siempre escasos y complicados. Esto podría sugerir un subregistro de pacientes, o bien que los ingresados allí ya han superado la fase aguda de presentación de arritmias y por tanto decrece su prevalencia en estos centros.

Otra evidencia en favor de esto último procede del Registro del Instituto de Cardiología y Cirugía Cardiovascular (ICCCV) ${ }^{17}$, a sólo $2 \mathrm{~km}$ del HHA. Su media de atención asciende a 7.2 pacientes por mes de estudio y su frecuencia de PCR fue del $9 \%$, muy inferior en el subgrupo de pacientes que recibió intervencionismo coronario primario respecto de los sujetos trombolizados $(4.7 \%$ vs. $11.7 \%, p=0.01)$. En el informe no se incluyen estos datos para los 68 pacientes sin reperfusión, por lo que es ésta la única conclusión extrapolable a otros centros sin intervencionismo.

\section{Registro de Síndromes Coronarios Agudos (RESCUE)}

En el Hospital Camilo Cienfuegos, los datos del Registro de Síndromes Coronarios Agudos (RESCUE) ${ }^{18}$ mostraron una incidencia de muerte súbita intrahospitalaria de $8.8 \%$ para pacientes con IMAEST. Para este análisis se consideraron las arritmias ventriculares malignas (TV y FV), así como los pacientes con rotura cardíaca con actividad eléctrica sin pulso como forma de presentación. Si se incluyera a los pacientes con IMASEST en este análisis, la frecuencia de MS descendería hasta cerca del $6 \%$ porque en este subgrupo la incidencia de MS fue mucho menor $(2.8 \%, p=0.01)$. En este mismo centro, en un registro previo desde $2014^{19}$, se notificó un $8.6 \%$ de mortalidad por arritmias ventriculares y cerca del $4 \%$ de los pacientes con IMAEST egresados vivos las presentó en algún momento de su ingreso. La aplicación de tratamiento con trombolíticos en comparación con aquellos que no lo recibieron se comportó como un factor protector al reducir la probabilidad de MS casi a la mitad, aunque sin 
diferencia estadística (OR, 0.52; IC, 0.247-1.094; $p=0.08)$. Sin embargo, la aplicación de trombolíticos no se diferenció entre pacientes con muerte súbita y aquellos que no $(57.34 \%$ vs. $45 \%$; $p=0.27$ ) en la muestra del RESCUE ${ }^{18}$. La posible diferencia puede explicarse porque el informe del Chávez-Ganzález, et al. incluyó como pacientes con arritmias a los que las desarrollan en las primeras horas de evolución.

En el caso específico del ILG, aunque se alcanza relación significativa con un punto de corte establecido en 1,303 puntos, los resultados difieren de los registrados por un grupo del Cardiocentro de Villa Clara ${ }^{20}$. Primero, el punto de corte hallado en la muestra del RESCUE dista demasiado (cerca de 150 puntos) del descrito, lo que supone una heterogeneidad entre un centro y otro en los valores al hacer extensivo este marcador pronóstico y nunca se logra un área bajo la curva superior a 0.70 y sólo se llega a un 0.656 (IC, 0.54-0.77; p = 0.01), muy lejos del 0.79 (IC, 0.77-0.89) del grupo referido. Mucho más cerca de los resultados de este estudio se halla el informe ya presentado de Castro-Torres ${ }^{15}$, del mismo centro, quien registró un área de 0.716 (IC95\%, 0.519-0.913; $p=0.042$ ) y también una variabilidad entre instituciones; debe señalarse que el informe de Díaz-Benítez, et al. tiene como objetivo relacionar el ILG y las complicaciones intrahospitalarias, sin especificar su tipo.

En cuanto al índice polimorfo/linfocitos*glucemia, el RESCUE no recoge datos referidos al diferencial del conteo de leucocitos. A pesar de esto se puede inferir que a partir de una posible homogeneidad en la relación polimorfo/linfocitos, la cual oscila entre 1.7 y 2.2, el determinante final del índice polimorfo/linfocitos*glucemia es el valor de esta última. Sin embargo, el resultado obtenido (área, 0.68; IC, 0.56-0.79; $p=0.01$ ) no es muy superior al hallado con esta muestra para el ILG, dado que ambos índices usan parámetros comunes.

En ninguno de los artículos anteriores se describe el tiempo de presentación de estas arritmias, por lo que el establecimiento de un perfil de riego basado en el tiempo de presentación de éstas es imposible. En el RESCUE, el $50 \%$ de los pacientes fallecidos por arritmias ventriculares la presentó en el primer día y otro $30 \%$ en el segundo.

Por último, los resultados de las redes de atención cubanas no son decepcionantes, aunque algo alejados de los indicadores de informes foráneos, ya que los resultados de éstos provienen de registros en los que el tratamiento de elección es el intervencionismo coronario. Quizás el sesgo principal se encuentra en la elevada tasa de mortalidad extrahospitalaria de pacientes con SCA que favorece un descenso de la incidencia de pacientes con arritmias ventriculares en etapa intrahospitalaria.

\section{Conclusiones}

Se observa una gran heterogeneidad en las investigaciones sobre muerte súbita intrahospitalaria. En apariencia, ésta no dejará de ser en Cuba una de las principales causas de muerte de los pacientes en la fase aguda de un infarto de miocardio.

\section{Financiamiento}

Ninguno.

\section{Conflicto de intereses}

Los autores declaran no tener ningún conflicto de intereses.

\section{Responsabilidades éticas}

Protección de personas y animales. Los autores declaran que para esta investigación no se han realizado experimentos en seres humanos ni en animales.

Confidencialidad de los datos. Los autores declaran que en este artículo no aparecen datos de pacientes.

Derecho a la privacidad y consentimiento informado. Los autores declaran que en este artículo no aparecen datos de pacientes.

\section{Bibliografía}

1. Sanchís-Gomar F, Pérez-Quilis C, Leischik R, Lucía A. Epidemiology of coronary heart disease and acute coronary syndrome. Ann Transl Med. 2016;4(13):256. doi: 10.21037/atm.2016.06.33

2. Martos-Benítez FD. Mortalidad cardíaca intrahospitalaria: epidemiología y estrategias de prevención. CorSalud 2017;9(2):95-105.

3. Pang BJM, Green MS. Epidemiology of ventricular tachyarrhythmia. Any changes in the past decades? Herzschr Elektrophys. DOI 10.1007/ s00399-017-0503-5.

4. Priori SG, Blomström-Lundqvist C, Mazzanti A. 2015 ESC guidelines for the management of patients with ventricular arrhythmias and the prevention of sudden cardiac death. Europace 2015;17(11):euv319.

5. Bière L, Niro M, Pouliquen H, Gourraud JB, Prunier F. Risk of ventricular arrhythmia in patients with myocardial infarction and non-obstructive coronary arteries and normal ejection fraction. World J Cardiol. 2017;9(3): 268-276.

6. Yousuf O, Chrispin J, Tomaselli GF, Berger RD. Clinical management and prevention of sudden cardiac death. Circ Res. 2015;116:2020-2040. DOI: 10.1161/CIRCRESAHA.116.304555.

7. Albanese M, Alpaslan K, Ouarrak T, Merguet P, Schneider S, Schöls W. In-hospital major arrhythmias, arrhythmic death and resuscitation after successful primary percutaneous intervention for acute transmural infarction: a retrospective single-centre cohort study. BMC Cardiovascular Disorders (2018) 18:116 https://doi.org/10.1186/s12872-018-0851-z.

8. Ibánez B, James S, Agewall S, Antunes MJ, Bucciarelli-Ducci Ch. 2017 ESC Guidelines for the management of acute myocardial infarction in patients presenting with ST-segment elevation. European Heart Journal 2017;00:1-66. doi:10.1093/eurheartj/ehx393.

9. Armas-Rojas NB, Dueñas-Herrera, de la Noval-García R, Ortega-Torres YY, Acosta-González M, Morales-Salinas A. Cardiopatía isquémica en Cuba. Una puesta al día. 2015. RCCCCV 2015;21(3). 
Arch Cardiol Mex. 2020;90(3)

10 Ochoa-Montes LA, González-Lugo M, Tamayo-Vicente ND, Gómez de Haz HJ, Correa-Azahares DP. Epidemiología de la muerte súbita cardíaca. Rev Cubana Hig Epidemiol. [Internet]. 2012 50(1):14-24. Available in http://scieloprueba.sld.cu/scielo.php?script=sci_arttext\&pi$d=S 1561-30032012000100003 \&$ Ing $=e s$.

11 Tamayo-Vicente ND, Ochoa-Montes LA, Vilches-Izquierdo E, González-Lugo M, García-Ones D. Infarto miocárdico crónico como factor de riesgo de muerte súbita cardíaca. Rev Cub Inv Biomed. 2015;34(2):145-156.

12. López-Ramírez M, Ramos-Emperador C, Gómez-Fernández M, Pe ña-Fernández NE, Fusté-Pedroso W. Factores relacionados con la muerte súbita en pacientes con infarto agudo de miocardio. CorSalud 2017;9(2):70-79.

13. Martínez-García G, Ravelo-Dopico R. Complicaciones intrahospitalarias del infarto del miocardio con elevación del segmento ST. Revista Cubana de Medicina Militar 2016;45(3):332-343.

14. Castro-Hernández FM, Alonso-Mariño NL, Ramírez-Gómez JI, Alonso-Mariño OL. Doble antiagregación plaquetaria en pacientes con infarto agudo de miocardio con elevación del segmento ST y tratamiento trombolítico. CorSalud 2016;8(4):217-226.

15. Castro-Torres Y, Yar-Khan N, Carmona-Puerta R. Nuevos marcadores de arritmias ventriculares malignas en pacientes con infarto agudo de miocardio con elevación del segmento ST. Resúmenes de trabajos pre- sentados al Concurso Internacional por el Premio «Néstor Acosta Tieles In Memoriam» del II Simposio Nacional de Muerte Súbita Cardiovascular y I Convención Iberoamericana de Muerte Súbita Cardiovascular (6-9 de diciembre de 2016, La Habana, Cuba). CorSalud 2017;9(3):174-179.

16. Sánchez-Ábalos VM, Bosch-Costrafeda C, Sánchez-Ábalos TM, González- Blanco JC. Morbilidad y mortalidad por infarto agudo del miocardio. MEDISAN 2014;18(4):516.

17. Martínez-Carrillo A, Sainz González-de la Peña BA, Ramos Gutiérrez B, Pacheco-Álvarez E, Zorio Suárez-BY, Castañeda-Rodríguez G. Infarto agudo con elevación del ST en el servicio de urgencias del Instituto de Cardiología. RCCCCV 2017;23:1.

18. Rodríguez-Ramos MA. Muerte súbita cardiovascular intrahospitralaria e infarto agudo de miocardio con elevación de ST: resultados de RESCUE. CorSalud 2017:9(4):255-262.

19. Chávez-González E, Rodríguez-Jiménez AE, Moreno-Martínez FL. Duración y dispersión del QRS para predecir arritmias ventriculares en las fases iniciales del infarto agudo de miocardio. Med Intensiva 2017;41(6):347-355. doi: 10.1016/j.medin.2016.09.008

20. Díaz-Benítez RE, Correa-Morales AM, Reyes-Hernández LM, Carvajal-Sánchez PA, González-Rivera EM, Coronado-Herrera Y. Hemoglobina glucosilada e índice leucoglucémico como determinaciones pronósticas en el síndrome coronario agudo. CorSalud 2016;8(3):153-163. 